\title{
QUEDAS ENTRE IDOSOS ATENDIDOS EM UMA UNIDADE BÁSICA DE SAÚDE DA AMAZÔNIA OCIDENTAL BRASILEIRA
}

Eder Ferreira de Arruda ${ }^{1}$, Ana Paula de Carvalho Castro ${ }^{2}$, Thays Nayara Pessoa dos Santos $^{2}$, Carminha Batista da Silva Bino², Rafael Tavares Lima |zel ${ }^{2}$

1. Docente do Centro Universitário UNINORTE, Rio Branco-AC, Brasil. E-mail: ederarrud@gmail.com.

2. Bacharel em Enfermagem pelo Centro Universitário UNINORTE, Rio Branco-AC, Brasil.

Recebido em: 15/05/2020 - Aprovado em: 15/06/2020 - Publicado em: 30/06/2020 DOI: 10.18677/EnciBio_2020B41

\begin{abstract}
RESUMO
O envelhecimento é marcado pela diminuição da capacidade funcional e o aumento de doenças e outros agravos, sobretudo as quedas. Objetivou-se identificar a frequência de quedas e de fatores relacionados entre idosos atendidos em uma unidade de saúde de Rio Branco-Acre. Realizou-se um estudo descrito observacional, de corte tipo transversal, realizado com 62 idosos por meio da aplicação de um questionário sobre as características sociodemográficas e fatores relacionados a quedas em idosos. Observou-se que a maioria dos idosos era do sexo feminino $(61,3 \%)$, com mais de 70 anos de idade $(53,2 \%)$, tinha o ensino fundamental (56,5\%), sem companheiro $(69,4 \%)$, com renda familiar de um salário mínimo $(62,9 \%)$ e residia na zona urbana do município (95,2\%). A frequência de quedas nos últimos 12 meses foi de $53,2 \%$, sendo que a maior parte escorregou $(57,6 \%)$, caiu três vezes ou mais $(60,6 \%)$, no interior do domicílio $(72,7 \%)$ e não apresentou complicações de saúde após o agravo $(57,6 \%)$. Dessa forma, a frequência de quedas entre os idosos foi elevada e reforça a necessidade de ações e estratégias de promoção da saúde voltadas a redução dos fatores preditores das quedas.
\end{abstract}

PALAVRAS-CHAVE: Acidentes por quedas; Atenção primária à saúde; Serviços de saúde para idosos.

\section{FALLS AMONG ELDERLY CARE IN A BASIC HEALTH UNIT IN THE WESTERN BRAZILIAN AMAZON}

\begin{abstract}
Aging is marked by a decrease in capacity and an increase in diseases and other conditions, mainly as falls. Objective: to identify the frequency of falls and related factors among elderly people seen at a health unit in Rio Branco-Acre. A crosssectional, observational, described study was carried out with 62 elderly people, applying a questionnaire on sociodemographic characteristics and factors related to falls in the elderly. He observed that the majority of the elderly were female $(61.3 \%)$, over 70 years old $(53.2 \%)$, had primary education $(56.5 \%)$, without a partner $(69.4 \%)$, income family with a minimum wage $(62.9 \%)$ and residence in the urban area of the municipality $(95.2 \%)$. The frequency of falls in the last 12 months was $53.2 \%$, with most of them slipping (57.6\%), falling three times or more $(60.6 \%)$, without a home $(72.7 \%)$ and with no health complications after the injury (57.6\%). Thus, the frequency of falls among the elderly was high and reinforced the need for health promotion actions and strategies aimed at reducing the factors that predict falls.
\end{abstract}

KEYWORDS: Fall accidents; Primary health care; Health services for the elderly. 


\section{INTRODUÇÃO}

Mundialmente, a população idosa tem crescido significativamente no decorrer das últimas décadas, principalmente nos países em desenvolvimento (OLIVEIRA; MENEZES, 2018). O aumento da população idosa se configura como um desafio às políticas públicas que precisam levar em consideração os direitos e as necessidades específicas de atenção à saúde destes indivíduos (DUARTE et al., 2018).

O processo de envelhecimento se caracteriza por intensas transformações biológicas e psicossociais que refletem diretamente nas condições e qualidade de vida dos idosos em virtude da diminuição da capacidade funcional e o aumento de doenças e outros agravos, sobretudo o risco de quedas (GASPAROTTO; FALSARELLA; COIMBRA, 2014).

As causas das quedas nos idosos podem ser classificadas em intrínsecas (condição física, postura, doenças ou efeitos de fármacos) e extrínsecas (ambiente doméstico não adaptado, falta de iluminação, uso rotineiro de tapetes e escadas, entre outros), sendo que cerca de 60 a $70 \%$ das quedas na terceira idade ocorrem em suas próprias residências e este percentual pode ser ainda maior (GUERRA et al., 2017; FERREIRA et al., 2016).

Dentre as consequências das quedas, durante essa fase da vida, destacamse: a redução da capacidade de realização das atividades da vida diária (AVD'S), hospitalizações e óbitos. As quedas podem ser evitadas através de medidas preventivas que priorizem a prática de exercícios físicos regulares, dieta balanceada, orientação adequada dos pacientes e seus cuidadores sobre os fatores de risco e através de correções dos fatores ambientais (TAKO et al., 2017).

As quedas nos idosos são um importante problema de saúde pública, devido às graves consequências que podem causar aos acometidos. Assim, é fundamental conhecer a frequência e os fatores relacionados a este agravo na terceira idade com a finalidade de estabelecer medidas de prevenção e promoção da saúde. Portanto, o objetivo desse estudo foi identificar a frequência de quedas e de fatores relacionados entre idosos atendidos em uma unidade de saúde de Rio Branco-Acre.

\section{MATERIAIS E MÉTODOS}

Trata-se de um estudo observacional descritivo, de corte transversal, com abordagem quantitativa envolvendo 62 idosos. Foi realizado em uma unidade de saúde de Rio Branco-AC, Brasil, composta por equipe multidisciplinar completa e que possui uma ampla área de abrangência. A escolha do local se deu em função de se tratar de unidade escola de referência para a instrumentalização da prática de acadêmicos.

O município de Rio Branco, capital do estado do Acre, está localizado na Amazônia Sul Ocidental (latitude: 958'26"S; longitude: 6748'27'O), possui uma área territorial de $8.834,942 \mathrm{~km}^{2}$ e população estimada em 407.319 habitantes no ano de 2019 (IBGE, 2020).

Para o cálculo amostral foi considerado média mensal de idosos atendidos no primeiro semestre de 2019, cujo total de atendimentos no ano foi de 324 indivíduos, sendo que o intervalo de confiança foi de $95 \%$ e um percentual de margem de erro de $5 \%$, o que determinou uma amostragem mínima de 62 idosos convidados para participarem do estudo.

Foram selecionados aqueles com idade mínima de 60 anos, cadastrados e frequentadores do grupo de idosos da unidade e os que voluntariamente aceitaram participar das atividades e ações propostas assinando o Termo de Consentimento 
Livre Esclarecido (TCLE). Por sua vez, foram excluídos os que não tinham condições físicas e cognitivas para responder o questionário.

A pesquisa foi desenvolvida nos meses de agosto e setembro de 2019 e foram coletados dados referentes às características sociodemográficas dos participantes (sexo, faixa etária, escolaridade, situação conjugal, renda familiar e zona de moradia) e aspectos relacionados às quedas (quantidade, local, motivo e consequências).

Os dados foram digitados e revisados no programa Microsoft $\$$ Office Excel e analisados no programa estatístico Statistical Package for the Social Sciences (SPSS), versão 21.0, no qual foram calculadas as frequências absolutas e relativas para as variáveis de interesse. A pesquisa foi submetida ao Comitê de Ética em Pesquisa do Centro Universitário UNINORTE e aprovado com o parecer $\mathrm{n}^{\circ}$ 3.441.983.

\section{RESULTADOS E DISCUSSÃO}

Conforme a tabela 1, a maioria dos participantes era do sexo feminino $(61,3 \%)$. De forma semelhante, no estudo realizado por Sousa et al. (2018) no município de Benevides (PA) se verificou que a maior parte dos idosos também era do sexo feminino $(57,7 \%)$.

Mundialmente, as mulheres são a maioria da população, possuem expectativa de vida superior à masculina, caracterizam-se por serem mais preocupadas com o autocuidado e por buscarem mais os serviços de saúde (CRUZ et al., 2020). Em contrapartida, os homens são patriarcalmente educados para serem avessos ao autocuidado e possuem dificuldade em procurar serviços que contemplem a sua saúde (BOTTON; CÚNICO; STREY, 2017).

Contudo, o sexo feminino está mais vulnerável às quedas, pois a partir da menopausa e com o avanço da idade as mulheres sofrem importantes alterações fisiológicas que associadas a comorbidades comuns na terceira idade aumentam 0 risco de cair (ESQUENAZI; SILVA; GUIMARÃES, 2014).

No que diz respeito à faixa etária, 53,2\% dos idosos estavam acima de 70 anos (Tabela 1). Da mesma forma, Sturmer et al. (2017) ao desenvolverem uma pesquisa com idosos em Marau (RS) encontraram que a maioria dos participantes tinha acima de 70 anos de idade (73,7\%). Assim também, Pilger, Menon e Mathias (2011) identificaram que $83,3 \%$ dos idosos de Guarapuava (PR) tinham idade superior a 70 anos.

Com o avançar da idade, de modo geral, aumenta os episódios de quedas, devido, sobretudo, à redução da força muscular, menor quantidade de massa magra, perda de massa óssea e ocorrência de patologias que reduzem a capacidade física, como, por exemplo, as doenças neurológicas, que afetam o equilíbrio e a postura (CASTRO et al., 2015).

No que se refere à escolaridade, $56,5 \%$ dos entrevistados tinha cursado o ensino fundamental e 35,5\% não eram alfabetizados (Tabela 1). De igual modo, em um estudo realizado em Vitória da Conquista (BA) envolvendo idosos com risco de quedas foi identificado que $38,0 \%$ dos participantes tinham o ensino fundamental e $28,0 \%$ eram analfabetos (LIMA et al., 2017). Este achado corrobora com os resultados de uma pesquisa com idosos que sofreram quedas em Tangará da Serra (MT) no qual foi evidenciado que $50,9 \%$ dos entrevistados não tinham estudos e $41,4 \%$ relataram ter de um a três anos de estudo (GASPAR et al., 2018).

De acordo com Abreu et al. (2016), os episódios de quedas entre os idosos podem ainda ser associados a baixa escolaridade e a outros fatores 
socioeconômicos que contribuem para um estado de iniquidade social, causando consequências diretas à capacidade funcional destes indivíduos, visto que se preocupam menos com sua saúde e tem mais dificuldades de acesso a informações sobre medidas de prevenção.

TABELA 1 - Características sociodemográficas de idosos atendidos em uma unidade de saúde do município de Rio Branco-Acre, 2019.

\begin{tabular}{lcc}
\hline Variável & $\mathbf{N}$ & $\%$ \\
\hline Sexo & 24 & 38,7 \\
Masculino & 38 & 61,3 \\
Feminino & & \\
Faixa etária (anos) & 16 & 25,8 \\
$60-65$ & 13 & 21,0 \\
$66-70$ & 33 & 53,2 \\
$>70$ & & \\
Escolaridade & 22 & 35,5 \\
Não alfabetizado & 35 & 56,5 \\
Ensino Fundamental & 02 & 3,2 \\
Ensino Médio & 03 & 4,8 \\
Ensino Superior & & \\
Situação Conjugal & 19 & 30,6 \\
Com Companheiro & 43 & 69,4 \\
Sem Companheiro & & \\
Renda familiar mensal* & 01 & 1,6 \\
Sem renda & 12 & 19,4 \\
<1 SM & 39 & 62,9 \\
1 SM & 10 & 16,1 \\
\hline Total & 62 & $\mathbf{1 0 0 , 0}$ \\
\hline Nota:
\end{tabular}

Nota: *Salário Mínimo - SM (2019) = R\$ 998,00.

Com relação à situação conjugal, $69,4 \%$ dos entrevistados não tinham companheiro (Tabela 1). De forma semelhante, Pinho et al. (2011) ao avaliarem o risco de quedas entre idosos de João Pessoa (PB) verificaram que a maioria dos participantes não tinha companheiro (84,3\%). Assim como, no estudo realizado em Natal (RN) que também analisou as características dos idosos e o risco de quedas foi identificado que $50,0 \%$ dos entrevistados eram solteiros, divorciados e separados (SANTOS et al., 2015). Segundo Abrantes (2013), idosos sem companheiros podem ter uma maior ocorrência de quedas, pois o fato de não ter alguém para compartilhar mutuamente os cuidados com a saúde contribui para o maior risco de cair.

No que se refere à renda familiar, $62,9 \%$ dos participantes recebiam um salário mínimo (Tabela 1). De igual forma, na pesquisa desenvolvida por Guerra et al. (2017) sobre o risco de quedas entre idosos vinculados a uma unidade de saúde de Aparecida de Goiânia (GO) foi observado que 66,0\% dos entrevistados recebiam um salário mínimo. Corroborando com os resultados de Abreu et al. (2016) que identificaram que a maior parte dos idosos de Cuiabá (MT) que caíram recebiam até dois salários mínimos (73,7\%).

Indivíduos com baixa renda, como é o caso dos idosos deste estudo, enfrentam maior risco de quedas, visto que tem menos acesso aos serviços de 
saúde e estão expostos a precárias condições de moradia e infraestrutura deficiente que podem favorecer as quedas (PAIVA, LIMA, BARROS, 2020).

De modo geral, a baixa renda acompanhada de baixa escolaridade e idade avançada podem contribuir para a vulnerabilidade social e predispor a pessoa ao maior ocorrência de quedas. Portanto, o processo de saúde e doença não se relaciona somente com aspectos individuais, mas também com a interação entre fatores socioeconômicos e demográficos (SOUZA et al., 2019).

Entre os 62 dos idosos entrevistados, 53,2\% $(n=33)$ relataram terem sofrido algum episódio de queda nos últimos 12 meses. Este resultado se assemelha ao valor encontrado por Ferreira et al. (2019) em Natal-RN (56,4\%). Porém, difere das frequências obtidas por Lopes et al. (2019) em Luziânia- GO $(75,0 \%)$ e Sousa-Araújo et al. (2019) em Uberaba-MG (24,7\%), por terem verificado valores superiores e inferiores de quedas entre idosos, respectivamente.

TABELA 2 - Fatores relacionados a quedas entre idosos atendidos em uma unidade de saúde do município de Rio Branco-Acre, 2019.

\begin{tabular}{lcc}
\hline Variável & N & $\%$ \\
\hline Quantidade & 08 & 24,3 \\
Uma vez & 05 & 15,1 \\
Duas vezes & 20 & 60,6 \\
Três vezes ou mais & & \\
Local de ocorrência & 24 & 72,7 \\
Interior do domicílio & 06 & 18,2 \\
Ambiente externo ao domicílio & 03 & 9,1 \\
Interior do domicílio e ambiente externo & & \\
Motivo & 03 & 9,1 \\
Desequilíbrio & 19 & 57,6 \\
Escorregão & 03 & 9,1 \\
Tontura & 04 & 12,1 \\
Tropeço & 04 & 12,1 \\
Outros & & \\
Complicações de saúde após a queda & 19 & 57,6 \\
Não & 14 & 42,4 \\
Sim & 33 & $\mathbf{1 0 0 , 0}$ \\
\hline Total &
\end{tabular}

Os achados de um estudo de revisão sistemática da literatura com metaanálise envolvendo 37 estudos, com um total de 58.597 idosos brasileiros, identificou que a frequência de quedas nos últimos 12 meses no país foi de 27,0\% com estimativas significativamente mais altas de quedas em mulheres na faixa etária $\geq 80$ anos comparado com 60 a 69 anos (ELIAS FILHO et al., 2019).

As quedas são um dos maiores problemas que impacta a saúde do idoso, seja pelo número de lesões, o risco de perda da independência e autonomia, hospitalizações ou pelo aumento da demanda com cuidados e custos relacionados à saúde (FERRETTI; LUNARDI; BRUSCHI, 2013).

Com o avanço da idade os riscos de quedas aumentam proporcionalmente, devido ao aumento de perda de equilíbrio e alterações osteomusculares (ARRUDA et al., 2019). O sedentarismo associado à comorbidades é um relevante fator intrínseco relacionado às quedas entre os idosos, portanto a prática regular de 
atividade física pode ajudar na prevenção dos episódios de quedas, pois favorecem a melhoria da força muscular, coordenação e equilíbrio (OLIVEIRA et al., 2017).

Com base na tabela $2,60,6 \%$ dos idosos caíram três vezes ou mais no último ano. De forma similar, Sousa-Araújo et al. (2019) verificaram em Uberaba (MG) que o número de quedas variou de uma a 12 vezes no último ano, com média de duas quedas por idoso, sendo que $35,1 \%$ duas ou mais, ou seja, quedas recorrentes.

De forma geral, as quedas são prejudiciais à saúde do idoso. Porém, quando ocorrem de forma recorrente, elas possuem um significado ainda mais preocupante, pois podem levá-los ao declínio funcional e social, ou seja, gera déficits que impactam fisicamente e compromete aspectos psicossociais do indivíduo e de seus familiares (GASPAROTTO; FALSARELLA; COIMBRA et al., 2014; MAIA et al., 2011).

A recorrência de quedas entre os idosos reforça a importância da prevenção e da necessidade de compreensão dos fatores que levam o idoso a um novo episódio deste agravo, assim permitindo intervenções e atividades de educação efetivas para a diminuição destes eventos na comunidade (ABREU et al., 2016).

No que se refere ao local de ocorrência da queda, 72,7\% dos entrevistados afirmaram ter caído dentro do domicílio e 38,7\% caíram em ambiente externo (Tabela 2). De forma similar, Tako et al. (2017) ao realizarem um estudo em Lagarto (SE) evidenciaram que 54,4\% dos idosos caíram dentro da residência. Assim como, Abrantes et al. (2013) observaram que a maior parte das quedas ocorreram no próprio domicílio, principalmente, entre os idosos mais velhos.

$\mathrm{Na}$ pesquisa desenvolvida por Ferretti, Lunardi e Bruschi (2013) em Chapecó (SC) o local da residência com mais ocorrência de quedas foi o banheiro $(24,94 \%)$ e cozinha (18,25\%). Todavia, neste estudo não foram investigados os locais da moradia em que os idosos mais sofreram quedas.

As condições do ambiente doméstico, a quantidade e a distribuição dos cômodos e a arquitetura das residências, bem como a presença de obstáculos como: degraus, piso escorregadio, tapete solto e pouca iluminação são importantes fatores predisponentes às quedas (TEIXEIRA et al., 2019).

Em relação ao motivo da queda, $57,6 \%$ dos participantes informaram ter escorregado (Tabela 2). Resultado semelhante foi encontrado em um estudo realizado em Caxias (MA) no qual $77,4 \%$ das quedas ocorreram após o idoso escorregar (OLIVEIRA; BALICA, 2017).

As quedas entre idosos no ambiente doméstico ocorrem, sobretudo, devido à maioria as residências não apresentarem estruturas e utensílios adaptados que atendam as necessidades de locomoção e que minimizem risco de escorregões e tropeções e consequentes quedas, tais como: piso regular e antiderrapante, presença de barras de apoio e segurança e iluminação adequada (CAVALCANTE et al., 2015).

De forma positiva, a maioria dos idosos não apresentou problemas de saúde após sofrerem algum episódio de queda (57,6\%), conforme a tabela 1 . De forma diferente, no estudo realizado por Jahana e Diogo (2007), observaram que a maioria dos idosos após um episódio de queda teve medo de cair e perder a independência, transformando o idoso em uma pessoa ansiosa e frequentemente deprimida (75,3\%). Resultado diferente, também foi identificado por Fabrício et al. (2004) ao realizarem uma pesquisa na cidade de Ribeirão Preto-SP verificaram que $64,0 \%$ dos idosos que caíram tiveram como consequência a ocorrência de fraturas. 
Idosos que sofrem quedas tem medo de sofrer quedas recorrentes, ficam mais apreensivos e menos confiante, ocasionando uma diminuição nas atividades diárias e contribuindo para o aumento da inatividade e o declínio da capacidade funcional, aumentando o risco para futuras quedas (MATOS et al., 2018). O aumento de internações e da busca por serviços especializados em decorrência das quedas é outra consequência comum (ARAÚJO NETO et al., 2017).

\section{CONCLUSÕES}

A frequência de quedas entre os idosos foi elevada, sendo um evento recorrente, sobretudo entre as mulheres e os indivíduos com idade mais avançada. Assim, percebe-se a necessidade do estabelecimento de medidas de educação em saúde, voltadas aos idosos e seus familiares, que priorizem a prevenção e a redução dos riscos predisponentes a quedas, principalmente, dentro do domicílio.

Apesar da relevância dos aspectos observados neste estudo, não se pode excluir a presença de possível viés de memória dos idosos. Contudo, as frequências encontradas podem indicar para existência de importantes fatores de risco na comunidade e para orientar os serviços de saúde quanto às estratégias de prevenção da ocorrência de quedas entre os idosos.

\section{REFERÊNCIAS}

ABRANTES, K.S.M.; MENEZES, T.N.; FARIAS, M.C.A.D.; SILVA, M.I.L.; ROLIM, V.E. et al. Caracterização das quedas em idosos socorridos pelo serviço de atendimento móvel de urgência. ABCS Health Sciences, v.38, n.3, p.126-132, 2013. Disponível em: <http://dx.doi.org/10.7322/abcshs.v38i3.17>.

ABREU, D.R.O.M.; AZEVEDO, R.C.S; SILVA, A.M.C; REINERS, A.A.O; ABREU, H.C.A. Fatores associados à recorrência de quedas em uma coorte de idosos. Ciência \& Saúde Coletiva, v.21, n.11, p.3439-3446, 2016. Disponível em: <http://dx.doi.org/ 10.1590/1413-812320152111.21512015>.

ARAÚJO NETO, A.H.; PATRÍCIO, A.C.F.A.; FERREIRA, M.A.M.; RODRIGUES, B.F.L.; SANTOS, T.D. et al. Quedas em idosos institucionalizados: riscos, consequências e antecedentes. Revista Brasileira de Enfermagem, v.70, n.4, p.752-758, 2017. Disponível em: <http://dx.doi.org/10.1590/0034-7167-2017-0107>.

ARRUDA, G.T.; WESCHENFELDER, A.J.; STRELOW, C.S.; FROELICHM, M.A.; PIVETTA, H.M.F. et al. Risco de quedas e fatores associados: comparação entre idosos longevos e não-longevos. Fisioterapia Brasil, v.20, n.2, p. 156-161, 2019. Disponível em:<https://doi.org/10.33233/fb.v20i2.2279>.

BOTTON, A.; CÚNICO, S.D.; STREY, M.N. Diferença de gênero no acesso aos serviços de saúde: problematizações necessárias. Mudanças - Psicologia da saúde, v. 25, n.1, p.67-72, 2017. Disponível em: <http://dx.doi.org/10.15603/21761019/mud.v25n1p67-72>.

CASTRO, P.M.M.A.; MAGALHÃES, A.L.; CRUZ, A.L.C.; REIS, N.S.R.D. Testes de equilíbrio e mobilidade funcional na predição e prevenção de riscos de quedas em idosos. Revista Brasileira de Geriatria e Gerontologia, v.18, n.1, p. 129-140, 2015. Disponível em: <http://dx.doi.org/10.1590/1809-9823.2015.13208>. 
CAVALCANTE, D.P.M.; SILVA, L.J.; MATOS, N.; BORGES, I.; ARAÚJO, D.P.A. et al. Perfil e ambientes de idosos, que sofreram quedas, atendidos em um ambulatório de Geriatria e Gerontologia no Distrito Federal. Revista Kairós Gerontologia, v.18, n.1, p. 93-97, $2015 . \quad$ Disponível em: <https://revistas.pucsp.br/kairos/article/view/23890/17135>.

CRUZ, P.K.R.; VIEIRA, M.A.; CARNEIRO, J.A.; COSTA, F.M.; CALDEIRA, A.P. Dificuldades do acesso aos serviços de saúde entre idosos não institucionalizados: prevalência e fatores associados. Revista Brasileira de Geriatria e Gerontologia, v.23, n.6, p.1-13, 2020. Disponivel em: <https://doi.org/10.1590/198122562020023.190113>.

DUARTE, G.P.; SANTOS, J.L.F.; LEBRÃO, M.L.; YEDA, A.O.D. Relação de quedas em idosos e os componentes de fragilidade. Revista Brasileira de Epidemiologia, v.21, suppl.2, p.1-9, 2018. Disponível em: <https://doi.org/10.1590/1980549720180017.supl.2>.

ELIAS FILHO, J.; BOREL, W.P.; DIZ, J.B.M.; BARBOSA, A.W.C; BRITTO, R.R; FELÍCIO, D.C. Prevalence of falls and associated factors in community-dwelling older Brazilians: a systematic review and meta-analysis. Cadernos de Saúde Pública, v. 35, n.8, p.1-16, 2019. Disponível em: <http://dx.doi.org/10.1590/0102311X00115718>.

ESQUENAZI, D.; SILVA, S.R.B.; GUIMARÃES, M.A.M. Aspectos fisiopatológicos do envelhecimento humano e quedas em idosos. Revista HUPE, v.13, n.2, p.11-20, 2014. Disponível em: <http://dx.doi.org/10.12957/rhupe.2014.10124>.

FABRÍCIO, S.C.C.; RODRIGUES, R.A.P.; JUNIOR, M.L.C. Causas e conseqüências de quedas de idosos atendidos em hospital público. Revista de Saúde Pública, v.38, n.1, p.93-99, 2004. Disponível em: <https://doi.org/10.1590/S0034$89102004000100013>$.

FERREIRA, L.M.B.M.; JEREZ-ROIG, J.; ANDRADE, F.L.J.P.; OLIVEIRA, N.P.D.; ARAÚJO, J.R.T.A. et al. Prevalência de quedas e avaliação da mobilidade em idosos institucionalizados. Revista Brasileira de Geriatria e Gerontogia, v.19, n.6, p. 9951003, 2016. Disponível em: <http://dx.doi.org/10.1590/1981-22562016019.160034>.

FERREIRA, L.M.B.M.; RIBEIRO, K.M.O.B.F.; JEREZ-ROIG, J.; ARAÚJO, J.R.T.; LIMA, K.C. Quedas recorrentes e fatores de risco em idosos institucionalizados. Ciência \& Saúde Coletiva, v.24, n.1, p.67-75, 2019. Disponível em: <http://dx.doi.org/10.1590/1413-81232018241.35472016>.

FERRETTI, F.; LUNARDI, D.; BRUSCHI, L. Causas e consequências de quedas de idosos em domicílio. Fisioterapia em Movimento, v.26, n.4, p.753-762, 2013. Disponível em: <https://doi.org/10.1590/S0103-51502013000400005>.

GASPAR, A.C.M.; SILVA, J.F.G.; MENDES, P.A.; FERREIRA, L.V.C.; AZEVEDO, R.C.S. et al. Perfil sociodemográfico e condições de saúde dos idosos que sofreram quedas. Journal of Research: Fundamental Care Online, v.10, n.4, p.1070-1076, 2018. Disponível em: <http://dx.doi.org/10.9789/2175-5361.2018.v10i4.1070-1076>. 
GASPAROTTO, L.P.R.; FALSARELLA, G.R.; COIMBRA, A.M.V. As quedas no cenário da velhice: conceitos básicos e atualidades da pesquisa em saúde. Revista Brasileira de Geriatria e Gerontologia, v.17, n.1, p.201-209, 2014. Disponível em: <https://doi.org/10.1590/S1809-98232014000100019>.

GUERRA, H.S.; BERNARDES, D.C.F.; SANTANA, J.A.; BARREIRA L.M.; NEVES, C.M. Avaliação do risco de quedas em idosos da comunidade. Revista Saúde.com, v.13, n.2, p.879-886, $2017 . \quad$ Disponível em: <http://periodicos2.uesb.br/index.php/rsc/article/view/462>

INSTITUTO BRASILEIRO DE GEOGRAFIA E ESTATISTICA. Rio Branco (AC). 2020. Disponível em: <https://cidades.ibge.gov.br/brasil/ac/rio-branco/panorama>.

JAHANA, K.O.; DIOGO, M.J.D. Quedas em idosos: Principais causas e consequências. Saúde Coletiva, v.04, n.17, p.148-153, 2007. Disponível em: <https://www.nescon.medicina.ufmg.br/biblioteca/imagem/1981.pdf>

LIMA, F.F.O.; FERREIRA, J.B.; REIS, L.A.; SANTOS, K.T.; LIMA, L.S. et al. Perfil Sociodemográfico e nível de dependência funcional de idosos com risco de quedas. Id On Line Revista Multidisciplinar e de Psicologia, v.11, n. 39, p.164-178, 2017. Disponível em: <https://idonline.emnuvens.com.br/id/article/view/985>.

LOPES, D.F.; SANTOS, S.D.; SOUZA, S.A.N.; SILVA, E.G.; SANTOS, W. L. Fatores relacionados a quedas em idosos. Revista de Iniciação Científica e Extensão, v.2, n.3, $2019.131-138, \quad$ Disponível em: $<$ https://revistasfacesa.senaaires.com.br/index.php/iniciacaocientifica/article/view/247>.

MAIA, B.C.; VIANA, P.S.; ARANTES, P.M.M.; ALENCAR, M.A. Consequências das quedas em idosos vivendo na comunidade. Revista Brasileira de Geriatria e Gerontologia, v.14, n.2, p.381-393, 2011. Disponível em: <http://dx.doi.org/10.1590/S1809-98232011000200017>.

MATOS, F.S.; JESUS, C.S.; CARNEIRO, J.A.O.; COQUEIRO, R.S.; FERNANDES, M.H. et al. Redução da capacidade funcional de idosos residentes em comunidade: estudo longitudinal. Revista Ciência e Saúde Coletiva, v.23, n.10, p. 3393-3401, 2018. Disponível em: <https://doi.org/10.1590/1413-812320182310.23382016>.

OLIVEIRA, D.V.; ANTUNES, M.D.; JÚNIOR LEITE, D.; LEME, D.E.C.; NASCIMENTO JÚNIOR, J.R.A. Associação do índice de massa corpora, força muscular e nível de atividade física de idosas praticantes de hidroginástica. Revista da Universidade Vale do Rio Verde, Três Corações, v.15, n.1, p. 417-427, 2017. Disponível em: <http://dx.doi.org/10.5892/ruvrd.v15i1.3352>.

OLIVEIRA, A.L.B.; MENEZES, T.M.O. Significado da religião/religiosidade para a pessoa idosa. Revista Brasileira de Enfermagem Reben, v.71, supl.2, p.823-829, 2018. Disponível em: <https://doi.org/10.1590/0034-7167-2017-0120>. 
OLIVEIRA, F.B.M.; BALICA, B.C.S. Prevalência de quedas e fatores associados em idosos. Revista Eletrônica Acervo Saúde, v. sup.5, p.249-255, 2017. Disponível em: <https://www.acervosaude.com.br/doc/S-5_2017.pdf>.

PAIVA, M.M.; LIMA, M.G.; BARROS, M.B.A. Desigualdades sociais do impacto das quedas de idosos na qualidade de vida relacionada à saúde. Ciência \& Saúde Coletiva, v.25, n.5, p.1887-1895, 2020. Disponível em: <http://dx.doi.org/10.1590/1413-81232020255.34102019>.

PILGER, C.; MENON, M.H.; MATHIAS T.A.F. Características sociodemográficas e de saúde de idosos: contribuições para os serviços de saúde. Revista LatinoAmericana de Enfermagem, v. 19, n5, 2011. Disponível em: <https://doi.org/10.1590/S0104-11692011000500022>.

PINHO, T.A.M.; SILVA, A.O.; TURA, L.F.R.; MOREIRA, M.A.S.P; GURGEL, S.N. et al. Avaliação do risco de quedas em idosos atendidos em Unidade Básica de Saúde. Revista da Escola de Enfermagem da USP, v.46, n.2, p. 320-327, 2011. Disponível em: <https://doi.org/10.1590/S0080-62342012000200008>.

SANTOS, R.K.M.; MACIEL, A.C.C.; BRITTO, H.M.J.S.; LIMA, J.C.C.; SOUZA, T.O. Prevalência e fatores associados ao risco de quedas em idosos adscritos a uma Unidade Básica de Saúde do município de Natal, RN, Brasil. Ciência \& Saúde Coletiva, v.20, n.12, p. 3753-3762, 2015. Disponível em: <https://doi.org/10.1590/1413-812320152012.00662015>.

SOUSA, F.J.D.; GONÇAVES, L.H.T.; PASKULIN, L.G.M.; GAMBA, M.A. Perfil sociodemográfico e suporte social de idosos na atenção primária. Revista de Enfermagem UFPE online, v.12, n.4, p.824-831, 2018. Disponível em: $<$ http://dx.doi.org/10.5205/1981-8963-v12i4a22855p824-831-2018>.

SOUSA-ARAÚJO, I.V.; GOMES, N.C.; SANTOS-NASCIMENTO, J.; RIBEIRO, C. C.N.R. TAVARES, D.M.S. Queda entre idosos: preditores e distribuição espacial. Revista de Salud Pública, v.21, n.2, p.187-194, 2019. Disponível em: $<$ http://dx.doi.org/10.15446/rsap.v21n2.70298>.

SOUZA, A.Q.; PEGORARI, M.S.; NASCIMENTO, J.S.; OLIVEIRA, P.B.; TAVARES, D.M.S. Incidência e fatores preditivos de quedas em idosos na comunidade: um estudo longitudinal. Ciência \& Saúde Coletiva, v.24, n.9, p. 3507-3516, 2019. Disponível em: <http://dx.doi.org/10.1590/1413-81232018249.30512017>.

STURMER, J.; BETTINELI, L.A.; AMARAL, P.P.; BORTOLUZZI, E.C.; DORING, M. Perfil sociodemográfico e clínico de idosos usuários das estratégias de saúde da família. Revista de Enfermagem UFPE online, v.11, supl.8, p. 3236-3242, 2017. Disponível em:<https://periodicos.ufpe.br/revistas/revistaenfermagem/article/view/110189/22075 $>$.

TAKO, K.V.; ANDRADE, L.C.; MARINHO, H.M.L.; NEVES, V.S.; SANTOS, A.E. et al. Perfil e prevalência de quedas em idosos. Revista de Enfermagem UFPE online, v.11, supl.11, p. 4687-4691, 2017. Disponível em: < https://periodicos.ufpe.br/revistas/revistaenfermagem/article/view/231210/25216>. 
TEIXEIRA, D.K.S.; ANDRADE, L.M.; SANTOS, J.L.P.; CAIRES, E.S. Quedas em pessoas idosas: restrições do ambiente doméstico e perdas funcionais. Revista Brasileira de Geriatria e Gerontologia, v.22, n.3, p.1-10, 2019. Disponível em: <http://dx.doi.org/10.1590/1981-22562019022.180229>. 